\title{
Efeito da música na modulação autonômica da frequência cardíaca em cardiopatas
}

\author{
Effect of music on the autonomic modulation of heart rate in cardiac patients \\ Efecto de la música sobre la modulación autónoma de la frecuencia cardíaca en pacientes cardíacos
}

Recebido: 20/09/2021 | Revisado: 26/09/2021 | Aceito: 28/09/2021 | Publicado: 02/10/2021

Victoria Ribeiro Pires

ORCID: https://orcid.org/0000-0002-3921-4415

Universidade Federal do Triângulo Mineiro, Brasil

E-mail: vribeiropires@gmail.com

Fernando Seiji da Silva

ORCID: https://orcid.org/0000-0002-6373-8817

Universidade Federal do Triângulo Mineiro, Brasil

E-mail: fernando.silva@uftm.edu.br

Marilita Falângola Accioly

ORCID: https://orcid.org/0000-0002-9623-3145

Universidade Federal do Triângulo Mineiro, Brasil

E-mail: marilita.accioly@uftm.edu.br

Joilson Meneguci

ORCID: https://orcid.org/0000-0003-2268-3589 Universidade Federal do Triângulo Mineiro, Brasil E-mail: joilson.meneguci@uftm.edu.br

Ana Paula Espindula

ORCID: https://orcid.org/0000-0002-9282-4482 Universidade Federal do Triângulo Mineiro, Brasil E-mail: ana.espindula@uftm.edu.br

Luciana Duarte Novais Silva

ORCID: https://orcid.org/0000-0002-1200-5680 Universidade Federal do Triângulo Mineiro, Brasil

E-mail: luciana.duarte.silva@uftm.edu.br

\begin{abstract}
Resumo
A música ocasiona diferentes respostas fisiológicas, algumas delas são alterações na pressão arterial, na frequência cardíaca e respiratória. Na literatura pesquisada foram encontrados poucos estudos que avaliam os efeitos da música na modulação autonômica cardíaca, sobretudo em pacientes cardiopatas ativos. Assim, o objetivo do presente estudo foi avaliar a modulação autonômica da frequência cardíaca sob o efeito de estímulo musical, por meio da análise da variabilidade da frequência cardíaca pelos métodos lineares e não-lineares. Metodologia: Participaram do estudo 11 pacientes cardiopatas ativos. Os voluntários permaneceram, antes do início do teste, em repouso por cinco minutos e 30 segundos e foram expostos a quatro músicas com ritmos diferentes, de forma aleatória, durante cinco minutos e 30 segundos cada uma; com um intervalo de 15 segundos entre elas. Foram analisados os índices lineares e não lineares, estatísticos e geométricos no domínio do tempo e os índices no domínio da frequência. Para análise dos dados foi realizado o teste de Friedman, com post-teste de Bonferroni, com nível de significância de 5\%. Resultados: Foram encontrados valores médios de batimentos cardíacos repetitivos significativamente maiores $(p<0,05)$ durante a música "Send my love" quando comparado ao repouso. Foi observado que não foram encontrados valores estatisticamente significantes nos diferentes espectros de potência, na razão BF/AF e nos índices não-lineares, quando comparadas as diferentes exposições musicais e ao repouso. Conclusão: Os índices de variabilidade de frequência cardíaca não foram alterados pela exposição musical nessa população estudada.
\end{abstract}

Palavras-chave: Musicoterapia; Sistema Nervoso Autônomo; Sistema Cardiovascular; Cardiopatia.

\begin{abstract}
Music causes different physiological responses, some of which are changes in blood pressure, heart rate and respiratory rate. In the researched literature, few studies were found that assess the effects of music on cardiac autonomic modulation, especially in active cardiac patients. Thus, the aim of the present study was to evaluate the autonomic modulation of heart rate under the effect of musical stimulation, through the analysis of heart rate variability by linear and non-linear methods. Methodology: Eleven active heart disease patients participated in the study. The volunteers remained, before the beginning of the test, at rest for five minutes and 30 seconds and were exposed to four songs with different rhythms, at random, for five minutes and 30 seconds each; with an interval of 15 seconds between them. The linear and non-linear, statistical and geometric indices in the time domain and the indices in the frequency domain were analyzed. For data analysis, the Friedman test was performed, with post-test Bonferroni, with a significance level of 5\%. Results: Mean values of repetitive heart beats were found significantly higher $(\mathrm{p}<0.05)$ during the song "Send my love" when compared to rest. It was observed that no statistically significant values were found in the different powers pectra,
\end{abstract}


in the $\mathrm{BF}$ ratio/AF and in non-linear indices, when comparing different musical exposures and at rest. Conclusion: The heart rate variability indices were not altered by musical exposure in this studied population.

Keywords: Music therapy; Autonomic Nervous System; Cardiovascular system; Heart disease.

\begin{abstract}
Resumen
La música provoca diferentes respuestas fisiológicas, algunas de las cuales son cambios en la presión arterial, la frecuencia cardíaca y la frecuencia respiratoria. En la literatura investigada, se encontraron pocos estudios que evaluaran los efectos de la música sobre la modulación autónoma cardíaca, especialmente en pacientes cardíacos activos. Así, el objetivo del presente estudio fue evaluar la modulación autónoma de la frecuencia cardíaca bajo el efecto de la estimulación musical, mediante el análisis de la variabilidad de la frecuencia cardíaca por métodos lineales y no lineales. Metodología: Participaron en el estudio once pacientes con cardiopatía activa. Los voluntarios permanecieron, antes del inicio de la prueba, en reposo durante cinco minutos y 30 segundos y fueron expuestos a cuatro canciones con diferentes ritmos, al azar, durante cinco minutos y 30 segundos cada una; con un intervalo de 15 segundos entre ellos. Se analizaron los índices lineales y no lineales, estadísticos y geométricos en el dominio del tiempo y los índices en el dominio de la frecuencia: componente de baja frecuencia (BF), alta frecuencia (AF) y la relación (BF / AF). Para el análisis de los datos se realizó la prueba de Friedman, con Bonferroni post-teste, con un nivel de significancia del 5\%. Resultados: Los valores medios de latidos repetitivos se encontraron significativamente más altos ( $\mathrm{p}<0.05)$ durante la canción "Envía mi amor" en comparación con el descanso. Se observó que no se encontraron valores estadísticamente significativos en los diferentes espectros de potencia, en la relación BF/AF y en índices no lineales, al comparar diferentes exposiciones musicales y en reposo. Conclusión: Los índices de variabilidad de la frecuencia cardíaca no se vieron alterados por la exposición musical en esta población estudiada.
\end{abstract}

Palabras clave: Terapia musical; Sistema nervioso autónomo; Sistema cardiovascular; Enfermedad del corazón.

\title{
1. Introdução
}

A música está entre os mais antigos traços cognitivos dos seres humanos, provavelmente devido à possibilidade de se expressar, da regulação das emoções e pelo prazer proporcionado ao ouvi-lá (Zatorre\&Salimpoor, 2013).

A musicoterapia tem sido utilizada como forma terapêutica para facilitar, promover e contribuir na qualidade de vida dos pacientes. De acordo com alguns autores, os elementos musicais fornecem um apoio emocional e espiritual, e com isso, consegue reduzir os níveis de estresse e ansiedade, sendo que muitos estudos trazem a música como forma adicional a outras terapias (cervellin \& lippi, 2011; Iwanaga, kobayashib \& kawasaki, 2005).

Nesse cenário, estudiosos puderam observar que o efeito da música ocasiona diferentes respostas fisiológicas, como alterações na pressão arterial, na frequência cardíaca e respiratória, na temperatura corporal, nas respostas galvânicas da pele, nos parâmetros bioquímicos do sistema endócrino e nas variações emocionais (Seijiat al., 2021; Zanini et al., 2009; Hatem, Lima \& Mattos, 2006).

O sistema nervoso autônomo (SNA) tem influências tônicas e reflexas sobre os fundamentais elementos do sistema cardiovascular, tais como frequência cardíaca (FC), pressão arterial (PA), resistência vascular periférica (RVP) e débito cardíaco (DC) (Hautala et al., 2003). A modulação autonômica do coração está relacionada estreitamente à FC, que sofre oscilações fisiológicas a cada batimento. Esse controle acontece pelas vias aferentes, medulares e vagais; no qual o sistema nervoso central recebe a informação, modulando a resposta; voltando ao coração pelas fibras eferentes: vagais rápidas e simpáticas lentas (Task, 1996).

Os ramos eferentes simpático e parassimpático, do sistema nervoso autônomo do coração, regula e modula as respostas e as oscilações da FC (Longo, Ferreira \& Correia, 1995). A variabilidade da frequência cardíaca (VFC) é definida como as oscilações dos intervalos entre batimentos cardíacos consecutivos (intervalos RR), sendo principalmente dependente da regulação extrínseca da FC. Bem como, a VFC retrata a capacidade do coração de se moldar à certas eventualidades, reagindo de forma ativa aos inesperados estímulos (Acharya et al., 2006.

A manifestação de eventos cardíacos, retratam um significativo motivo de diminuição na VFC, em indivíduos teoricamente saudáveis (Rebelo et al., 2013; Neves et al., 2006; Ribeiro et al., 2001), cardiopatas (Kunzat al., 2012; Novais et al., 2004; Kleiger et al., 1987) bem como obesos (Paschoal, Trevizan \& Scodeler, 2009), pessoas com diabetes tipo 2 
(Menezes, Moreira \& Daher, 2004) e hipertensão arterial sistêmica (HAS) (Schmid, Neumann \& Brugnara, 2003); sendo essa atenuação relativa a uma diminuição da atividade vagal ou aumento da simpática (Menezes, Moreira \& Daher, 2004; Task, 1996).

A VFC pode ser analisada por dois métodos:_lineares e não-lineares, e se por um acaso ocorrem mudanças nesses padrões, é possível inferir adaptações na modulação autonômica anormal e prejuízos à saúde (Carvalho et al., 2011; Vanderlei et al., 2009). A análise da VFC, tanto em indivíduos sadios ou com doenças, demonstra ser um método de acessível aplicabilidade e alcance dos dados, com um bom prenunciador de doenças e agravos em estados patológicos já presentes (Lopes et al., 2009), ao mesmo tempo que estudos anteriores indicaram que a musicoterapia tem efeitos benéficos sobre a VFC em pacientes enfermos (Chuang et al., 2011; Chuang et al., 2010).Cardiopatas ativos são indivíduos que aderem a programas de reabilitação cardíaca (RC) e que, com isso, apresentam inúmeras mudanças hemodinâmicas, metabólicas, miocárdicas, vasculares, alimentares e psicológicas que estão associadas ao melhor controle dos fatores de risco e à melhora da qualidade de vida (Diretrizes de reabilitação cardíaca, 2005). A RC é o conjunto de intervenções que garantem melhores condições físicas, psicológicas e sociais de pacientes com doenças cardiovasculares pós-aguda e crônicas, fazendo a reintegração do indivíduo na sociedade, por meio do comportamento saudável com intuito de minimizar ou reverter à progressão da doença (Fletcher et al., 2013).

Atualmente, existem poucos trabalhos na literatura (Seiji et al., 2021; Zanini et al., 2009; Hatem, Lira \& Mattos, 2006) que comprovam os benefícios trazidos pela música na população como um todo, sendo que na população específica desse estudo, de cardiopatas ativos, a literatura é ainda mais escassa, justificando a proposta de realização desde estudo. Assim, nossa hipótese é que a música interfere na VFC em indivíduos cardiopatas. Desta maneira, o objetivo do estudo foi avaliar a VFC pelos métodos lineares e não-lineares, sob o efeito de estímulo musical em indivíduos cardiopatas que realizam RC.

\section{Metodologia}

Estudo de abordagem quantitativa, observacional e transversal (Pereira et al., 2018), desenvolvido no Laboratório de Cardiologia respiratória, no Centro de Reabilitação Prof. Dr. Fausto da Cunha Oliveira da Universidade Federal do Triângulo Mineiro (UFTM), aprovado pelo Comitê de Ética em Pesquisa da UFTM sob parecer de no 3.013.931.

Todos os participantes foram informados sobre os procedimentos experimentais aos quais seriam submetidos, do caráter não invasivo dos testes, bem como no fato destes não afetarem sua saúde. Foi também esclarecido quanto ao sigilo das informações colhidas durante a realização do trabalho, resguardando suas identidades. Os indivíduos que concordaram em participar assinaram o termo de consentimento livre e esclarecido, de acordo com as normas do Conselho Nacional de Saúde $(466 / 12)$.

Participaram do estudo 11cardiopatas, selecionados por conveniência, atendidos no estágio do curso de Fisioterapia da UFTM, com idade média de 66 anos ( \pm 9,7 anos); 72,7\% dos indivíduos eram do sexo masculino, e 27,3\% feminino; 54,5\% apresentaram infarto agudo do miocárdio, 45,5\% eram hipertensos; $18,2 \%$ diabéticos, e 9,1\% apresentam obesidade e/ou doença arterial coronariana, ressaltando que alguns indivíduos possuíam mais de uma comorbidade (Tabela 1). 
Tabela 1: Variáveis demográficas dos 11cardiopatas.

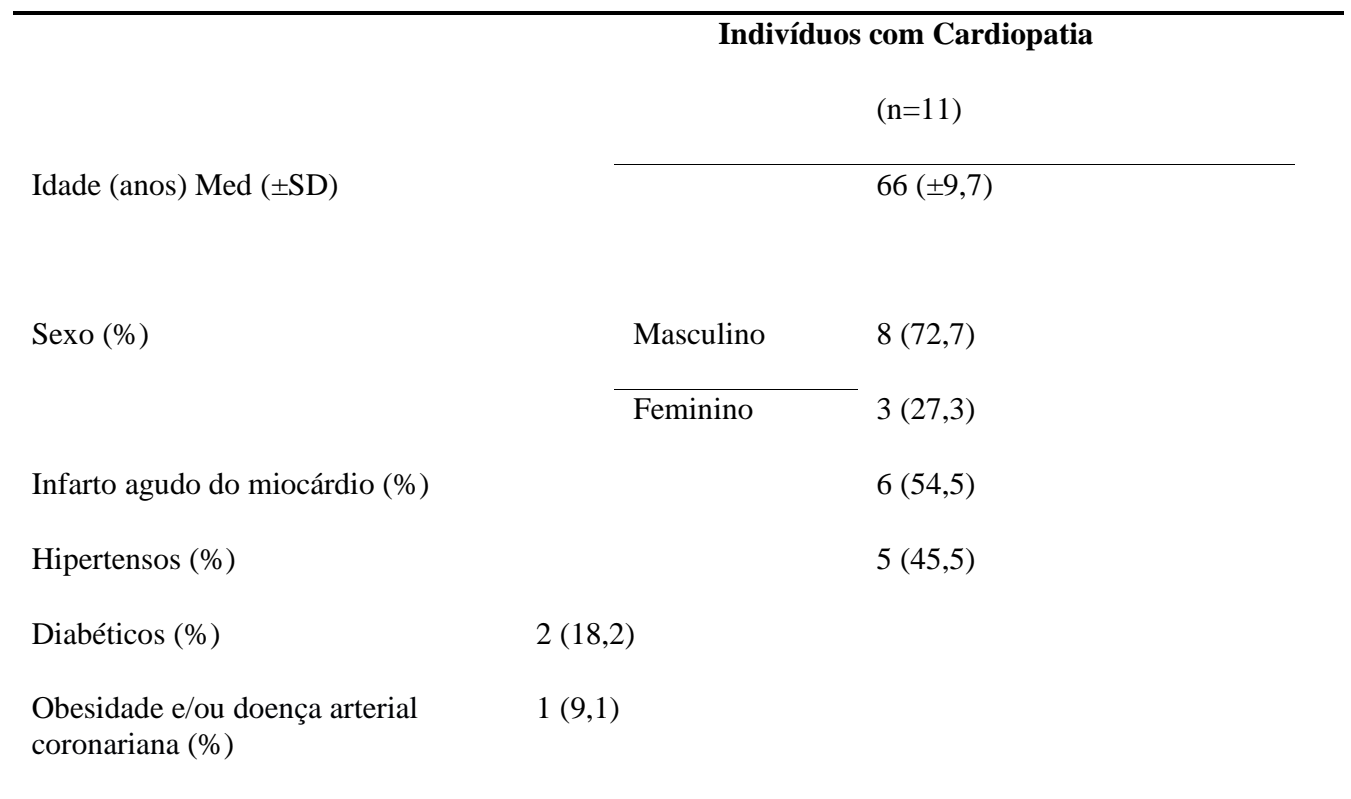

Med: média; ( $($ SD): desvio padrão da média; $(\%)$ :porcentagem; n: número. Autores.

Como critério de inclusão foi estabelecido que os cardiopatas fossem atendidos há mais de seis meses no setor de Reabilitação Cardíaca do Centro de Reabilitação Prof. Dr. Fausto da Cunha Oliveira, e que nunca deveriam ter realizado qualquer tipo de treinamento musical. Foram excluídos os voluntários que apresentassem diagnósticos ou relato de distúrbios auditivos como por exemplo: perdas auditivas parciais ou total, alterações no labirinto ou algum outro tipo de comprometimento que pudesse afetar a audição; fumantes ou qualquer impedimento pessoal.

Foi recomendado aos participantes a comparecerem com roupas e calçados confortáveis, que não fizessem o uso de bebidas alcoólicas e/ou estimulantes 24 horas antes dos testes, realizassem refeição leve com mínimo de duas horas antes do teste e não realizassem atividade física extenuante no dia anterior.

A preparação dos equipamentos, dos materiais e a organização da sala foram realizados com uma hora de antecedência à chegada de cada participante. Para reduzir a ansiedade e expectativa por parte dos participantes, foram feitos procedimentos de familiarização com o protocolo de teste, com a equipe e equipamentos. Os protocolos foram realizados sempre pelos mesmos avaliadores, sendo mantido um trânsito mínimo de pessoas no laboratório durante a execução dos experimentos.

Este teste teve como objetivo avaliar a modulação autonômica da resposta da frequência cardíaca a partir de sua variabilidade (VFC) durante o repouso em silêncio e sob estímulo musical. Para isso, foi utilizado um monitor de frequência cardíaca, da marca POLAR, modelo RS800CX para obtenção dos dados de FC e posterior análise da VFC. Esse equipamento é composto de uma cinta (onde estão localizados os eletrodos) e um relógio de pulso (onde é feita a gravação dos dados de FC e de sua variabilidade). Primeiramente foi feito o posicionamento da cinta no tórax (na altura do coração) e do relógio no pulso do voluntário, além do fone de ouvido. A partir de então, os voluntários foram orientados a manter-se em repouso na posição sentada, com uma respiração tranquila e evitando conversar com os avaliadores.

Após 10 minutos, para estabilização das variáveis cardiorrespiratórias, foi iniciada a coleta dos dados de frequência cardíaca de repouso por cinco minutos e 30 segundos, na posição sentada, em uma sala com o ambiente silencioso. Depois desse período, os voluntários foram expostos a um período de 22 minutos de música, com um intervalo de um minuto entre cada música, mantendo a mesma coleta de FC. Foram impostas quatro músicas com ritmos diferentes, de forma aleatória, durante cinco minutos e 30 segundos, sendo as seguintes músicas: 1)Photograph, de Ed Sheeran; música instrumental 
percussão, ritmo lento; 2) Hello, de Adele; música pop, vozes, ritmo lento; 3) Castle on the hill, de Ed Sheeran; música pop, ritmo rápido; 4) Send my Love, de Adele; música pop indie, vozes, ritmo rápido. Todas adquirida via Itunes.

Após a coleta da FC, foram descartados os 15 segundos iniciais e finais de cada amostra, a fim de selecionar trechos mais estáveis de sinal. Seguido por uma inspeção visual da distribuição dos dados de FC coletados, para verificar a estabilidade do traçado obtido, bem como a ausência de artefatos. Os dados fornecidos pelo software Polar Precision Performance $S w$, foram anexados ao programa Kubios HVR Standard para análise dos métodos lineares e não-lineares.

Os métodos lineares são divididos em: análise no domínio do tempo, os quais mensuram cada intervalo RR durante determinado intervalo de tempo, baseando-se em métodos estatísticos, pelos índices: RR médio (média de intervalos RR); SDNN (desvio padrão de todos os intervalos RR normais, em milissegundos [ms]); RMSSD (raiz quadrada da média do quadrado das diferenças ao quadrado entre intervalos RR normais adjacentes, em ms) e o pNN50 (porcentagem dos intervalos $\mathrm{RR}$ adjacentes com diferença de duração maior que $50 \mathrm{~ms}$ ). E pelo método geométrico, o índice triangular, que é formado a partir de uma elaboração de um histograma de densidade de intervalos RR normais, contendo no eixo horizontal (eixo x), os comprimentos dos intervalos RR e, no eixo vertical (eixo y) as frequências em que ocorreram. Sendo que, a união das colunas configura um triangulo, visto que a base desta figura pronuncia-se sobre a variabilidade dos intervalos RR (Gonzaga et al., 2019; Vanderlei et al., 2009).

Por meio da análise espectral, domínio da frequência, foram obtidas as bandas de baixa frequência (BF), e de alta frequência (AF). Foi utilizado duas faixas de frequência que melhor representassem a atuação dos componentes vagal e simpático no controle da FC, ou seja, faixa $\mathrm{BF}$, correspondendo de 0,04 a $0,15 \mathrm{~Hz}\left(\mathrm{~ms}^{2}\right)$, que corresponde a ação tanto do componentes vagal quanto do simpático sobre o coração, porém com predomínio do simpático;e a faixa de AF, que corresponde de 0,15 a $0,4 \mathrm{~Hz}\left(\mathrm{~ms}^{2}\right)$, que fala à respeito da modulação respiratória, sendo um informativo da atuação do nervo vago sobre o coração. Além dos valores absolutos de $\mathrm{BF}$ e $\mathrm{AF}$, tais componentes são expressos como a razão entre as áreas absolutas de baixa e alta frequência (razão BF/AF), que é indicativo do balanço simpato vagal(Vanderlei et al., 2009).

Os métodos não-lineares explanam sobre o comportamento dos fenômenos biológicos, tratando-se de um bom preditor de morbimortalidade no âmbito clínico. Dentre os métodos não-lineares utilizados para análise da VFC, podemos citar: análise de flutuações depuradas de tendências (DFA), usado para quantificar as propriedades de escala fractal dos sinais de intervalo RR de intervalo curto $(\alpha 1)$ e intervalo longo ( $\alpha 2)$; análise do plot de Poincaré (elipse da correlação entre intervalos RR consecutivos), calculado de forma quantitativa, os três índices: SD1 (ms) (desvio padrão da variabilidade instantânea, batimento a batimento), SD2(ms) (desvio padrão dos intervalos RR contínuos em longo prazo) e a razão SD1/SD2 (razão das variações curta e longa dos intervalos RR); a entropia aproximada (ApEn) que faz a medição do grau de irregularidade e complexidade em uma série temporal experimental, visto que quanto maior for o valor da ApEn, maior será a complexidade; e a entropia da amostra (SamPen) que é uma medida da desordem existente na série, com valores maiores para indivíduos saudáveis e valores menores em cardiopatas (Madeiro et al., 2011; Ferreira et al., 2010; Vanderlei et al., 2009; Acharya et al., 2006; Brunetto et al., 2005) .

Para análise dos dados foram realizados os procedimentos de estatística descritiva, distribuição da frequência, cálculo de medida de tendência central e de dispersão. Para comparar os efeitos dos estilos musicais na VFC foi utilizado o Teste de Friedman, com comparação múltipla de médias de ordens com correção de Bonferroni, sendo a normalidade dos dados testada previamente pelo teste de Shapiro-Wilk. O nível de significância adotado foi inferior a 5\% ( $\mathrm{c}<0,05)$. A análise estatística foi efetuada com o software Statistical Package for Social Sciences (SPSS), versão 24. 


\section{Resultados}

Em relação ao gosto musical, $100 \%$ dos cardiopatas disseram que gostaram das músicas de forma geral. A ordem das preferências musicais foi para as músicas "Send my love" (45,5\%) e "Hello" (27,3), seguido das músicas "Castle on the hill" $(18,2 \%)$ e Photograph (9\%).

Na tabela 2, são apresentados os resultados em relação à análise linear no domínio do tempo. Os valores médios de RR foram maiores durante a exposição à música "Send my love", quando comparado ao repouso ( $\mathrm{p}=0,022)$. Foi observado maiores valores de SDNN durante a exposição musical "Castlle on the hill" e na variável RMSSD durante a música "Photograph", sem significância estatística (Tabela 2).

Nos diferentes espectros de potência foi possível verificar uma tendência a significância, porém com diferença não estatística, nos valores de BF durante a exposição à música "Castle on the hil" quando comparado ao repouso (p=0,06), como demonstrado na tabela 3 que descreve a análise linear no domínio da freqüência (Tabela 3 ).

$\mathrm{Na}$ análise não-linear da variável DFA, $\alpha 2$ foi observado tendência a significância, porém com diferença não significativa,nas músicas "Castlle on the hill" e "Send my love" comparadas ao repouso (p=0,059) (Tabela 4). O valor de SD1 foi maior durante a música "Photograph", e os índices SD2, SD2/SD1 e DFA, $\alpha 1$ foram maiores durante a exposição musical de "Castlle on the hil", entretanto sem significância estatística (Tabela 4).

Tabela 2. Análise linear do domínio do tempo.

\begin{tabular}{|c|c|c|c|c|c|c|}
\hline & & Música 1 & Música 2 & Música 3 & Música 4 & \\
\hline & Repouso & & & & & Valor de p \\
\hline & & Photograph & Hello & Castlleonthehill & Sendmylove & \\
\hline & 900,00 & 903,00 & 894,00 & 892,00 & 912,00* & \\
\hline Mean RR & & & & & & $0,022^{\alpha}$ \\
\hline & $(850,00 ; 985,00)$ & $(846,00 ; 1.023,00)$ & $(873,00 ; 1.000,00)$ & $(836,00 ; 994,00)$ & $(858,00 ; 1.006,00)$ & \\
\hline & 15,10 & 13,00 & 13,40 & 16,30 & 15,60 & \\
\hline SDNN & & & & & & 0,424 \\
\hline & $(9,40 ; 22,00)$ & $(10,00 ; 26,70)$ & $(9,10 ; 28,70)$ & $(10,20 ; 36,10)$ & $(10,90 ; 22,00)$ & \\
\hline & 13,60 & 16,90 & 15,40 & 16,70 & 15,70 & \\
\hline RMSSD & & & & & & 0,382 \\
\hline & $(9,70 ; 27,00)$ & $(9,30 ; 39,00)$ & $(8,60 ; 36,80)$ & $(8,60 ; 39,60)$ & $(9,60 ; 23,90)$ & \\
\hline & 0,52 & 0,28 & 0,88 & 0,86 & 0,65 & \\
\hline pNN50 & & & & & & 0,243 \\
\hline & $(0,00 ; 7,55)$ & $(0,00 ; 11,07)$ & $(0,00 ; 18,01)$ & $(0,00 ; 19,49)$ & $(0,00 ; 4,23)$ & \\
\hline $\mathbf{R R}$ & 4,08 & 3,85 & 4,17 & 4,34 & 4,08 & \\
\hline $\begin{array}{l}\text { triangular } \\
\text { index }\end{array}$ & $(2,85 ; 5,26)$ & $(3,06 ; 8,24)$ & $(3,07 ; 9,41)$ & $(3,12 ; 6,55)$ & $(2,97 ; 5,47)$ & 0,373 \\
\hline
\end{tabular}

${ }^{\alpha}$ Nível de significância para "p" 5\%(p<0,05). * Significância comparado ao repouso. Fonte: Autores. 
Tabela 3: Análise linear no domínio da frequência

\begin{tabular}{|c|c|c|c|c|c|c|}
\hline & Repouso & Música 1 & Música 2 & Música 3 & Música 4 & Valor de $p$ \\
\hline & & Photograph & Hello & Castlle on the hill & Sendmylove & \\
\hline \multirow[t]{2}{*}{ BF } & 36,00 & 59,00 & 53,00 & 121,00 & 86,00 & 0,066 \\
\hline & $(22,00 ; 140,00)$ & $(29,00 ; 224,00)$ & $(29,00 ; 219,00)$ & $(27,00 ; 177,00)$ & $(58,00 ; 307,00)$ & \\
\hline \multirow[t]{2}{*}{$\mathbf{A F}$} & 38,00 & 85,00 & 61,00 & 86,00 & 62,00 & 0,611 \\
\hline & $(5,00 ; 101,00)$ & $(25,00 ; 421,00)$ & $(24,00 ; 370,00)$ & $(23,00 ; 438,00)$ & $(37,00 ; 171,00)$ & \\
\hline \multirow[t]{2}{*}{ BF/AF } & 25,00 & 23,00 & 35,00 & 29,00 & 34,00 & 0,845 \\
\hline & $(8,00 ; 60,00)$ & $(9,00 ; 100,00)$ & $(20,00 ; 58,00)$ & $(16,00 ; 55,00)$ & $(18,00 ; 81,00)$ & \\
\hline
\end{tabular}

${ }^{\alpha}$ Nível de significância para "p" 5\%(p<0,05). Fonte: dos autores.

Tabela 4: Análise não linear.

\begin{tabular}{|c|c|c|c|c|c|c|}
\hline & Repouso & $\begin{array}{c}\text { Música } 1 \\
\text { Photograph }\end{array}$ & Música 2 & Castlle on the hill & $\begin{array}{c}\text { Música } 4 \\
\text { Sendmylove }\end{array}$ & $\begin{array}{l}\text { Valor } \\
\text { de } p\end{array}$ \\
\hline \multirow[t]{2}{*}{ SD1 } & 9,70 & 12,00 & 10,90 & 11,80 & 11,10 & 0,379 \\
\hline & $(6,90 ; 19,100)$ & $(6,60 ; 27,60)$ & $(6,10 ; 26,00)$ & $(6,10 ; 28,00)$ & $(6,80 ; 16,90)$ & \\
\hline \multirow[t]{2}{*}{ SD2 } & 18,90 & 14,30 & 14,30 & 19,90 & 17,80 & 0,543 \\
\hline & $(12,40 ; 24,60)$ & $(12,30 ; 30,10)$ & $(12,20 ; 31,10)$ & $(13,30 ; 32,20)$ & $(14,20 ; 28,50)$ & \\
\hline SD2/SD1 & 1,65 & 1,42 & 1,63 & 1,68 & 1,55 & 0,624 \\
\hline \multirow[t]{2}{*}{ ApEn } & 1,14 & 1,16 & 1,11 & 1,12 & 1,13 & 0,396 \\
\hline & $(1,06 ; 1,18)$ & $(1,05 ; 1,19)$ & $(1,06 ; 1,14)$ & $(1,08 ; 1,15)$ & $(1,04 ; 1,17)$ & \\
\hline \multirow[t]{2}{*}{ SamPen } & 1,63 & 1,94 & 1,80 & 1,78 & 1,90 & 0,663 \\
\hline & $(1,36 ; 1,88)$ & $(1,66 ; 2,04)$ & $(1,59 ; 2,11)$ & $(1,51 ; 2,00)$ & $(1,63 ; 2,01)$ & \\
\hline DFA, $\alpha 1$ & 0,90 & 0,84 & 0,91 & 0,98 & 0,95 & 0,804 \\
\hline
\end{tabular}




\begin{tabular}{|c|c|c|c|c|c|}
\hline DFA, $\alpha 2$ & 0,49 & 0,50 & 0,51 & 0,33 & 0,33 \\
\hline & $(0,31 ; 0,62)$ & $(0,38 ; 0,64)$ & $(0,43 ; 0,65)$ & $(0,00 ; 0,43)$ & $(0,00 ; 0,43)$ \\
\hline
\end{tabular}

${ }^{\alpha}$ Nível de significância para "p" 5\%(p<0,05). Fonte: dos autores.

\section{Discussão}

O presente estudo teve como objetivo avaliar a VFC pelos métodos lineares e não-lineares, sob o efeito de estímulo musical, em indivíduos cardiopatas que realizavam reabilitação cardíaca. Tendo como hipótese que a música interfere na variabilidade da frequência cardíaca em indivíduos cardiopatas, sendo essa não validada.

Ultimamente, as pesquisas científicas descrevem sobre a relação existente entre estímulos musicais e sistema cardiovascular (Hanser, 2014). Evidências experimentais mostram que, a depender do estilo musical oferecido, as emoções evocadas terão um efeito com respostas variadas sobre a regulação da atividade cardíaca regional, FC, VFC, PA e frequência respiratória (FR) (Seiji et al., 2021; Koelsch \& Jancke, 2015). Em um estudo com 27 mulheres saudáveis entre 18 e 30 anos, que comparou a resposta aguda do sistema nervoso autônomo diante dos estímulos musicais clássico e heavy metal, durante 10 minutos, relatou maior valor durante a exposição à música do tipo clássica, porém sem diferença estatística (Etzel et al., 2006). Os resultados do presente estudo são discordantes da literatura apresentada, já que foi encontrado o valor médio de RR maior durante o estímulo musical de ritmo rápido. Entretanto, esta investigação avaliou indivíduos cardiopatas, que já podem apresentar algum grau de disfunção autonômica.

Em relação ao índice SDNN, o estudo de Etzel et al., (2006), com 18 indivíduos saudáveis expostos à 12 clipes de música, que induziam felicidade, tristeza ou medo, descreve maior valor no clipe que incentivava ao medo, do que em relação aos outros estímulos.

No estudo de Ferreira, Vanderlei \& Valenti (2015), foi observado aumento no índice BF (precursor perceptível da modulação simpática) e um decréscimo no índice AF (simbólico da modulação parassimpática, rápida retirada vagal) para a música heavy metal em comparação ao clássico. O estilo heavy metal, segundo Roque et al.,(2013), produz um estresse agudo e com isso é capaz de realizar alterações na modulação autonômica cardíaca. Em um estudo executado com estudantes sujeitos a ouvir três condições distintas de músicas (relaxante, excitante e nenhum estímulo musical), pode-se destacar que os índices AF e BF aumentaram nas músicas relaxantes e excitantes; com maiores valores de AF durante a música relaxante em comparação a excitante (Umemura \& Honda, 1998).Todavia, estes estudos descritos em anos com diferença de faixa de tempo de treze e quinze anos, não corroboram com o presente.

As bandas de AF e BF, também, foram relatadas na literatura, sendo a mais baixa durante o estímulo musical do que em silêncio; sendo que essa redução durante o estímulo musical reflete uma modulação de ambos os tônus (simpático e parassimpático) (Koelsch \& Jancke, 2015). Os resultados obtidos no presente estudo demonstraram inversão da dinâmica apresentada por esses autores, pois os menores valores de AF e BF, foram em repouso e não quando expostos a música.

De acordo com o estudo de Chuang et al(2011), que teve como objetivo avaliar se a música é capaz de melhorar a função autonômica de mulheres doentes com câncer de mama, os índices: RR, DPNN, RMSSD, AF e BF aumentaram significativamente os seus valores de T1 (antes da primeira sessão de terapia de música) e T2 (após a $4^{\text {a }}$ sessão) e de T1 a T3 (após $8^{\mathrm{a}}$ sessão); sendo que a $4^{\mathrm{a}}$ ocasião analisada, T4 (após o término do tratamento com estímulo musical) não foi observado diferenças. 
Em um estudo que avaliou a regulação autonômica cardíaca com diferentes estilos de estimulação auditiva musical (barroco e heavy metal) com mulheres saudáveis, descrevem que os índices SD1 e o SD2, ao ouvir dois estilos musicais, tendiam a uma redução em comparação ao grupo controle (Roque et al., 2013). No presente estudo, é possível observar que ambos os índices apresentaram maiores valores nas exposições musicais em indivíduos cardiopatas.

De acordo com os autores Urakawa e Yokoyama (2005), exercícios físicos ativam uma dominância simpática do sistema nervoso autônomo (SNA) (), e se o exercício físico for acompanhado de música, acentuaria essa dominância, promovendo uma excitação fisiológica, aumentando, consequentemente, a atividade fisiológica, além de tornar a realização de tal atividade mais prazerosa.

Segundo Nakamura et al., (2007), pode- se observar que quando os camundongos eram expostos a música; isso fazia com que se diminuísse a atividade simpática do nervo renal e a pressão arterial através da via auditiva, dos neurônios histamínicos e núcleo hipotalâmico supraquiasmático.

ConformeValenti et al.,(2012), a estimulação auditiva com música diminuiu a frequência cardíaca e a pressão arterial em humanos (Lee et al., 2005) e espontaneamente em ratos hipertensos (Sutoo \& Akiyama, 2004), indicando que a música pode afetar a função autonômica cardíaca.

Já Iwanaga \& Moroki(1999), estudaram alunos de graduação e sugerem que apesar da música favorita do indivíduo diminuir o estado de tensão, independentes do tipo de música, as respostas fisiológicas (frequência cardíaca, respiração e pressão arterial) serão maiores durante a música excitativa do que durante a música sedativa. Ou seja, a preferência musical não é capaz de afetar as respostas fisiológicas. Estes resultados indicam que o fator dominante que afeta a resposta emocional foi o tipo de música, não a preferência individual.

Em consoante, Nakahara et al.,(2009), que teve como objetivo em seu estudo investigar os efeitos das emoções evocadas pela música de piano na FC e na sua variabilidade em uma população de quatro mulheres e seis homens universitários fisicamente ativos e saudáveis, pode-se inferir que, quando os indivíduos foram induzidos a se expressar diante do estímulo musical com emoção, gerou melhores respostas do que a música sem emoção.

Índices de VFC foram também utilizados para demonstrar que músicas de alta intensidade e ruídos escutados por gravador, provocam modificações na modulação autonômica cardíaca, sendo que os estilos musicais como o clássico resultam em supressão do nervo simpático e músicas do estilo rock causam estímulo deste nervo (Umemura \& Honda, 1998).

Em um estudo com 54 indivíduos, que recebiam assistência ventilatória, pode-se observar que no grupo de música, se observou menores níveis de ansiedade, diminuição da frequência cardíaca e respiratória com o tempo, em comparação aos sujeitos do grupo controle; sendo que com um único tratamento de musicoterapia, se mostrou eficaz na resposta ao relaxamento (Chlan, 1998).

No estudo de Palakanis et al(1994), com pacientes submetidos a sigmoidoscopia flexível visando analisar o estado de ansiedade desses indivíduos, a musicoterapia se mostrou como um tratamento não farmacêutico seguro e eficaz para esses pacientes, quando se trata na redução do quadro de ansiedade.

No estudo de Nieto-Romero (2017), o efeito de musicoterapia sobre o nível de ansiedade do adulto cardiopata submetido à ressonância magnética, que teve como metodologia a seguinte intervenção: um grupo escutou música clássica e o outro, escutou músicas de sua preferência por 30 minutos antes da ressonância e, durante a realização do exame ( $\geq 20$ minutos). Foi verificado que a terapia com música reduziu a frequência cardíaca, a frequência respiratória e tensão arterial sistólica, em ambos os grupos; porém o nível de ansiedade teve maior redução no grupo de pacientes que escutaram as músicas de sua preferência.

Segundo o estudo de Mandel (2007), que testou a eficácia da musicoterapia na melhoria dos resultados relacionados à saúde de pacientes na fase II da RC, houve uma maior diminuição da pressão arterial sistólica no grupo da musicoterapia tanto 
pré como pós-tratamento; em relação as medidas psicológicas, algumas diferenças foram encontradas em quatro meses após o tratamento, tais como melhora na saúde geral e uma no funcionamento social.

Na revisão sistemática realizada por Bradt, Dileo \& Potvin(2013), com enfoque em indivíduos com doença arterial coronariana, a qual analisou o efeito das intervenções musicais usadas com o intuito de reduzir a ansiedade e o sofrimento e a melhorar o funcionamento fisiológico destes pacientes, os resultados indicaram que as intervenções musicais têm um pequeno efeito benéfico no sofrimento psíquico nessa população, sendo esse efeito consistente entre os estudos. Além disso, como resultados principais, a revisão aponta que o efeito musical consegue reduzir a frequência cardíaca, frequência respiratória e pressão arterial sistólica. Porém o estudo mostra que, em relação à variabilidade da frequência cardíaca e a depressão, não foram descobertas poderosas evidências.

Ao observara musicoterapia de uma perspectiva mais ampla, as evidências sugerem que a musicoterapia também possui influências em outros tipos de populações, em outros grupos de doenças, e além da associação do estímulo musical juntamente com outras terapias, e que o efeito produzido não só ocorre na questão da variabilidade da frequência cardíaca, mas também em vários outros sistemas corporais.

Este estudo apresenta como limitações o fato de a amostra ter sido selecionada por conveniência e heterogênea em relação às cardiopatias. Desta forma, sugere-se a ampliação de estudos sobre essa temática com um número maior de cardiopatas ativos, com seleção randomizada e com grupos homogêneos quanto a suas cardiopatias.

\section{Conclusão}

Por meio do presente estudo conclui-se que tanto pelos métodos, lineares e não-lineares, da variabilidade da frequência cardíaca em indivíduos cardiopatas ativos, os índices não foram alterados pela exposição musical, provavelmente devido à disfunção autonômica presente nessa população. Sugere-se para futuras pesquisas o aumento do número de participantes e análise em separado para os sexos, assim como aumento do número de exposições às musicas.

\section{Agradecimentos}

Os autores agradecem pelo apoio do Conselho Nacional de Desenvolvimento Científico e Tecnológico para a Melhoria de Pessoal de Nível Superior (CNPq), a Fundação de Ensino e Pesquisa do Estado de Minas Gerais (FAPEMIG), a Fundação de Ensino e Pesquisa de Uberaba (FUNEPU). Aos membros doLaboratório de Cardiologia respiratória, do Centro de Reabilitação Prof. Dr. Fausto da Cunha Oliveira da Universidade Federal do Triângulo Mineiro (UFTM) e aos indivíduos que participaram do estudo.

\section{Referências}

Acharya, R. U., Joseph, P. K., Kannathal, N., Lim, C. M., Suri, J. S. (2006). Heart rate variability: a review. Medical and Biological Engineering and Computing. 44(12): 1031-105.

Bradt, J., Dileo, C., Potvin, N. (2013). Music for stress and anxiety reduction in coronary heart disease patients. The Cochrane Collaboration. 1-104.

Brunetto, A. F., Silva, B. M., Roseguini, B. T., Hirai, D. M., Guedes, D. P. (2005). Limiar ventilatório e variabilidade da freqüência cardíaca em adolescentes. RevBrasMed Esporte. 11(1): 22-27.

Carvalho, T. D., Pastre, C. M., de Godoy, M. F., Ferreira, C., Pitta,F. O., de Abreu, L. C., Cipulo Ramos, E. M., Valenti, V. E., Marques Vanderlei, L. C. (2011). Fractal correlationpropertyofheart rate variability in chronicobstructivepulmonarydisease. Int J ChronObstructPulmDis. 6(6): 23-28.

Cervellin, G., Lippi, G. (2011). A journey with Euterpe. Sinfonia concertante for music, heart and brain. RecentiProg Med. 9(102):352-358.

Chlan, L. (1998). Effectiveness of a music therapy intervention on relaxation and anxiety for patients receiving ventilatory assistance. Heart Lung. 27(3):169176. 
Chuang, C. Y., Han, W. R., Li, P. C., Song, M. Y., Young, S. T. (2011). Effect of Long-Term Music Therapy Intervention on Autonomic Function in Anthracycline-Treated Breast Cancer Patients. IntegratCancerTher. 10(4):312-316.

Chuang, C. Y., Han, W. R., Li, P. C., Young, S. T. (2010). Effects of music therapy on subjective sensations and heart rate variability in treated cancer survivors: a pilot study. Complement Ther Med. 18(5):224-226.

Diretriz de Reabilitação Cardíaca. (2005). Arq. Bras. Cardiol.84(5): 431-440.

Etzel, J. A., Johnsen, E. L., Dickerson, J., Tranel, D., Adolphs, R. (2006). Cardiovascular and respiratory responses during musical mood induction. International Journal of Psychophysiology. 61: 57-69.

Ferreira, L. L., Vanderlei, L. C. M., Valenti, V. E. (2015). Resposta aguda do sistema nervoso autônomo a diferentes estímulos auditivos musicais em mulheres. RevNeurocienc. 23(3): 420-426.

Ferreira, M. T., Messias, M., Vandereli, L. C. M., Pastre, C. M. (2010). Caracterização do Comportamento Caótico de Variabilidade da Frequência Cardíaca (VFC) em Jovens Saudáveis. Tend. Mat. Apl. Comput. 11(2): 141-150.

Fletcher, G. F., Ades, P. A., Kligfield, P., Arena, R.., Balady, G. J., Bittner, V. A., Coke, L. A., Fleg, J. L., Forman, D. E., Gerber, T. C., Glati, M., Madan, K., Rhodes, J., Thompson, P. D., Williams, M. A. (2013). Exercise Standards for Testing and Training: a statement for healthcare professionals from the American Heart Association. Circulation. 128(8): 873-934.

Gonzaga, L. A., de Paulo, T. R. S., Viezel, J., Vanzella, L. M., Freitas Jr, I. F., Vanderlei, L. C. M. (2019). Alterações na Modulação Autonômica Cardíaca em mulheres com Câncer de Mama em Uso de Inibidores de Aromatase e sua Relação com Variáveis Bioquímicas. ArqBrasCardiol. 112(5): 555-563.

Hanser, S.B. (2014). Music Therapy in Cardiac Health Care. Cardiology in Review. 22(1):37-42.

Hatem, T., Lira, P., Mattos, S. (2006). The therapeutic effects of music in children following cardiac surgery. Jornal de Pediatria. 82(3): 186-192.

Hautala, A. J., Makikallio, T. H., Kiviniemi, A., Laukkanen, R. T., Nissila, S., Huikuri, H. V., Tulppo, M. P. (2003). Cardiovascular autonomicfunction correlates withthe response toaerobic training in healthysedentarysubjects. Am J Physiol Heart CircPhysiol. 285(4): 1747-1452.

Iwanaga, M., Kobayashib, A., Kawasaki, C. (2005). Heart rate variability with repetitive exposure to music. Biol Psychol. 70(1):61-66.

Iwanaga, M., Moroki,Y. (1999). SubjectiveandPhysiological Responses to Music StimuliControlled Over ActivityandPreference. JournalOfMusicTherapy. 36(1): $26-38$

Kleiger, R. E., Miller, J. P., Bigger Jr, J. T., Moss, A. J. (1987). Decreased heart rate variability and its association with increased mortality after acute myocardial infarction. The American Journal Of Cardiology. 59(4):256-262.

Koelsch, S., Jancke, L. (2015). Music and the heart. European Heart Journal. 36: 3043-3048.

Kunz, V. C., Borges, E. N., Coelho, R. C., Gubolino, L. A., Martins, L. E. B., Silva, E. (2012). Linear and nonlinear analysis of heart rate variability in healthy subjects and after acute myocardial infarction in patients. Braz J Med Biol Res. 45(5): 450- 458.

Lee, O. K., Chung, Y. F., Chan, M. F., Chan, W. M. (2005). Music and its effect on the physiological responses and anxiety levels of patients receiving mechanical ventilation: a pilot study. Journal Of Clinical Nursing. 14(5):609-620.

Longo, A., Ferreira, D., Correia, M. (1995) Variabilidade da frequência cardíaca. RevPortCardiol. 14(3):241-262.

Lopes, P. F. F., de Oliveira, M. I. B., André, S. M. S., Nascimento, D. L. A., Silva, C. S. S., Rebouças, G. M., Felipe, T. R., Albuquerque Filho, N. J. B., Medeiros, H,J. (2013). Aplicabilidade Clínica da Variabilidade da Frequência Cardíaca. RevNeuro cienc.21(4): 600-603.

Madeiro, J. P. V., Seisdedos, C. R. V., Cortez, P. C., Marques, J. A. L. (2011). Análise de Desempenho da Entropia Aproximada (ApEn) na análise da Variabilidade da Frequência Cardíaca (VFC).V Latin American CongressonBiomedicalEngineering CLAIB.16(21): 1182-1185.

Mandel, S. E., Hanser, S. B., Secic, M., Davis, B. A. (2007). Effects of music therapy on health-related outcomes in cardiac rehabilitation: a randomized controlled trial. J Music Ther. 44(3):176-197.

Menezes Júnior, A. S., Moreira, H. G., Daher, M. T. (2004). Análise da variabilidade da frequência cardíaca em pacientes hipertensos, antes e depois do tratamento com inibidores da enzima conversora da angiotensina II. ArqBrasCardiol. 83(2): 165-16.

Nakahara, H., Furuya, S., Obata, S., Masuko, T., Kinoshita, H. (2009). Emotion-related Changes in Heart Rate and Its Variability during Performance and Perception of Music. Ann N Y AcadSci. 1169(1):359-362

Nakamura, T., Tanida, M., Nijima, A., Hibino, H., Shen, J., Nagai, K. (2007). Auditory stimulation affects renal sympathetic nerve activity and blood pressure in rats. NeuroscienceLetters. 416(2):107-112.

Neves, V. F. C., Perpétuo, N. M., Sakabe, D. I., Catai, A. M., Gallo Jr, L., Silva de Sá, M.F., Martins, L.E.B., Silva, E. (2006). Análise dos índices espectrais da variabilidade da frequência cardíaca em homens de meia idade e mulheres na pós-menopausa. RevBras Fioter.10(4): 401-406.

Novais, L. D., Sakabe, D. I., Takahashi, A. C.M., Gongora, H., Taciro, C., Martins, L.E.B., Oliveira, L., Silva, E., Gallo Junior, L., Catai, A.M (2004).Avaliação da variabilidade da frequência cardíaca em repouso de homens saudáveis sedentários e de hipertensos e coronariopatas em treinamento físico. RevBrasFisioter. 8(3):207- 213.

Palakanis, K. C., De Nobile, J. W., Sweeney, W. B., Blankenship, C.L. (1994). Effect of music therapy on state anxiety in patients undergoing flexible sigmoidoscopy. Diseases of the Colon \& Rectum. 37(5):478-481. 
Research, Society and Development, v. 10, n. 12, e563101220831, 2021 (CC BY 4.0) | ISSN 2525-3409 | DOI: http://dx.doi.org/10.33448/rsd-v10i12.20831

Paschoal, M. A., Trevizan, P. F., Scodeler, N .F. (2009). Variabilidade da frequência cardíaca, lípides e capacidade física de crianças obesas e não-obesas. ArqBrasCardiol. 93(3): 239-246.

Pereira, A. S., Shitsuka, D. M., Parreira, F. J \&Shitsuka, R. (2018). Metodologia da pesquisa científica. [e-book]. Santa Maria. Ed. UAB/NTE/UFSM.https://repositorio.ufsm.br/bitstream/handle/1/15824/Lic_Computacao_Metodologia-Pesquisa-Cientifica.

Rebelo, A. C., Verlengia, R., Kunz, V. C., Tamburus, N. Y., Sá, M. F. S., Hirata, R. D. C., Cerda, A., Hirata, M. H., Silva, E. (2013). Association of estrogen receptor alpha gene polymorphisms with autonomic modulation of heart rate in users and nonusers of oral contraceptives. Contraception.88(1):183-188.

Ribeiro, T. F., Azevedo, G. D., Crescêncio, A. C., Marães, V. R. F. S., Papa, V., Catai, A. M., Verzola, R. M. M., Oliveira, L., Silva de Sá, M.F., Gallo Jr, L., Silva, E. (2001). Heart rate variabilityunderrestingconditions in postmenopausalandyoungwomen. Braz J MedBiol Res. 34(7):871- 877.

Romero, N. (2017). Efectos de la musicoterapia sobre elnivel de ansiedaddel adulto cardiópata sometido a resonancia magnética. Enferm. Univ. 14(2): 88-96.

Roque, J. M. A. (2009). Variabilidade da Frequência Cardíaca. Coimbra: Faculdade de Ciências do Desporto e Educação Física da Universidade de Coimbra,. Trabalho de Seminário integrado no plano de estudos do grau de Licenciatura em Educação Física.

Roque, A. L., Valenti, V. E., Guida, H. L., Campos, M. F., Knap, A., Vanderlei, L. C. M., Ferreira, C., Abreu, L. C. (2013). The effects of different styles of musical auditory stimulation on cardiac autonomic regulation in healthy women. Noise \& Health. A Bimonthly Inter-disciplinary International Journal. 15(65): 281-287.

Schmid, H., Neumann, C. A., Brugnara, L. (2003). O diabetes melito e a desnervação dos membros inferiores: a visão do diabetólogo. J Vasc Bras. 2(1):3748 .

Seiji , F., Novais, L. D., Custódio Júnior, J. da Silva., Henriques Júnior, S. Q., Espindula, A. P., Smith, R. L. (2021). Impact of music on autonomic modulation in different age groups. Journal Research, Society and Development. 10(8): e1310817030. DOI: 10.33448/rsd-v10i8.17030.

Sutoo, D., Akiyama, K. (2004). Music improves dopaminergic neurotransmission: demonstration based on the effect of music on blood pressure regulation. Brain Research. 1016(2): 255-262.

Task, F. (1996). Heart rate variability: standards of measurement, physiological interpretation and clinical use. European Heart Journal. (3): 354-381.

Umemura, M., Honda, K. (1998). Influence of music on heart rate variability and comfort--a consideration through comparison of music and noise. $J$ Hum Ergol. 27(1): 30-38

Urakawa, K., Yokoyama, K. (2005). Music can enhance exercise-induced sympathetic dominancy assessed by heart rate variability. The Tohoku J Exp Med. 206(3):213-218

Valenti, V. E., Guida, H. L., Frizzo, A. C. F., Cardoso, A. C. V., Vanderlei, L. C. M., Abreu, L. C. (2012). Auditory stimulation and cardiac autonomic regulation. Clinics. 67(8): 955-958.

Vanderlei, L. c.em., Pastre, C. M., Hoshi, R. A., Carvalho, T. D., Godoy, M. F. (2009). Noções básicas de variabilidade da frequência cardíaca e sua aplicabilidade clínica. RevBrasCirCardiovasc. 24(2): 205-217.

Zanini, C. R. O., Jardim, P. C..V., Salgado, C. M., Nunes, M. C., Urzêda, F. L., Carvalho, M. V. C., Pereira, D. A., Jardim, T. S. V., Souza, W. K, S. B. (2009). O Efeito da musicoterapia na qualidade de vida e na pressão arterial do paciente hipertenso. Arq. Bras. Cardiol. 93(5): 534-540.

Zatorre, R., Salimpoor, V. (2013). From perception to pleasure: music and its neural substrates. Proc Natl Acad Sci. $110(2)$ : $10430-10437$. 